\title{
A Novel SCMA Codebook Design Method Based on Low Error Probability Criteria
}

\author{
$1^{\text {st }}$ Yuan Zhang, $4^{\text {th }}$ Fucheng Guo \\ College of Electronic Science and \\ Technology \\ National University of Defense Technology \\ Changsha, China \\ zhangyuan@nudt.edu.cn, gfcly75@163.com
}

\author{
$2^{\text {nd }}$ Zilong Liu \\ School of Computer Science and Electronic \\ Engineering \\ University of Essex \\ Colchester, United Kingdom \\ zilong.liu@essex.ac.uk
}

\author{
$3^{\text {rd }}$ Pei Xiao \\ $5 G I C \& 6 G I C$, Institute for \\ Communication Systems (ICS) \\ University of Surrey \\ Guildford, United Kingdom \\ p.xiao@surrey.ac.uk
}

\begin{abstract}
In this paper, we propose a method to construct codebooks for downlink sparse code multiple access (SCMA) systems. Different from the traditional methods in which codebooks are designed for each user, we allocate codebooks for each subchannel based on the most common modulation QAM. Then, low error probability criteria to measure the SCMA codebook performance in AWGN channel and Rayleigh fading channel are proposed. And simulated annealing algorithm is used to optimize the codebooks based on the criteria. Simulation results prove that the proposed codebooks exhibit good performance in both Rayleigh fading channel and AWGN channel, especially for high order SCMA systems.
\end{abstract}

Keywords - Sparse code multiple access, codebook design, simulated annealing, AWGN channel, Rayleigh fading channel, highorder codebook.

\section{INTRODUCTION}

$5 \mathrm{G}$, the latest generation of cellular mobile communications, provides an access to satisfy the need of ultra-high definition video, car networking, internet of things (LoT), etc., which targets reduced latency, high data rate, larger system capacity and massive connectivity. The system is supposed to support 100 billion connections, $1 \mathrm{~ms}$ latency and data rate of several tens of megabits per second [1].

Traditionally, different users are distinguished by different resources that they occupy, such as CDMA, TDMA, and FDMA. However, limited resources cannot satisfy the need of increasing users. So non-orthogonal multiple access (NOMA) [2] schemes are proposed as possible solutions, which means in a given resource, more than one users are transmitted by introducing controllable interference and higher receiver complexity. Proposed NOMA techniques include three categories [3] : power-domain NOMA (PD-NOMA) [4, 5], code-domain NOMA (CD-NOMA) [6-8], and a combination of PD-NOMA and CD-NOMA called power domain sparse code multiple access [6]. Code-domain NOMA (CD-NOMA) supports the multiple transmissions by assigning different codewords to different users [9]. Inspired by low-density signature (LDS) [6], Huawei proposes a CD-NOMA technique called sparse code multiple access (SCMA) with low decoding complexity and good performance [10-12]. The design of codebook [10] is a virtual problem for SCMA.
Nikopour et al. [13] and Taherzadeh et al. [10] propose a multi-stage suboptimal method for multiuser SCMA codebooks design. First, design a mother constellation, and then, by allocating users' operators, each user obtains its own codebook. And the SCMA codebook is calculated by interleaving users' codebooks on transmitting sources. Based on the direction, some effective methods in [10, 14-18] for the design of downlink SCMA systems codebook are proposed. Cai et al. proposed a multi-dimensional codebook design based on constellation rotation and interleaving [14], which can be easily extended for any codebook and system parameters, such as number of sources, codebook size and codebook dimension. Yu et al. proposed a design method of SCMA codebooks based on star-QAM [15]. Based on golden angel modulation (GAM), Zeina et al. proposed a codebook design method with good performance [3].

In this paper, a novel method for SCMA codebook design is proposed. We develop a novel class of power-imbalanced SCMA codebooks which allocate different power to different users in downlink channel. Different from the traditional structure in which codebooks are designed for each user, we allocate codebooks for each sub-channel. And to achieve better performance, optimization criteria based on minimum Euclidean distance, minimum product distance and kissing number are proposed for AWGN and Rayleigh fading channels. Then, simulated annealing algorithm (SA) is adopted to optimization the constellation. Numerical simulation results show that our proposed codebooks lead to significantly improved bit error rates (BERs) over both Gaussian channels and Rayleigh fading channels, especially for high-order.

The rest of the paper is organized as follows. In Section II, SCMA system model is introduced briefly. Section III explains the new design method of SCMA codebooks in detail. Minimum bit error rate (BER) optimization criteria are explained in Section IV. And simulation results are shown in Section V. In Section VI, the paper is concluded and analyzed.

\section{SCMA SySTEM MODEL}

For a SCMA downlink system with multiple users and one base station, as is shown in Fig. 1, $J$ users' signals are sent by one antenna and transmitted simultaneously on $K(J>$ 


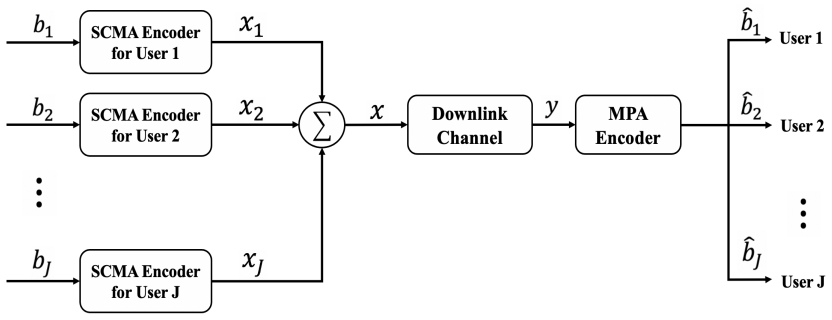

Fig. 1: System model for downlink SCMA

$K)$ resource elements (REs). Define the overloading factor $\lambda=J / K$ [13], which illustrates that $J$ users share $K$ orthogonal resources, e.g., $K$ orthogonal frequency subcarriers. Define $d_{f}$ as the number of users on each resource. Traditionally, in the orthogonal access techniques, e.g., OFDMA, TDMA, each user's signal transmits on one orthogonal RE exclusively, so $J$ is less or equal to $K$, which means lower resource efficiency.

SCMA encoder performs a mapping from $q=\log _{2}^{M}$ coded bits to a $K$-dimensional complex codebook with size $M$, which is defined as

$$
f: \mathbb{B}^{\log _{2}^{M}} \rightarrow \chi, \mathbf{x}=f(\mathbf{b})
$$

where $\mathbf{b}$ denotes the incoming channel coded bits and $K$ dimensional complex codeword $\mathrm{x} \in \chi \subset \mathbb{C}^{K}$ is a sparse vector with cardinality $\|\chi\|=M$.

In SCMA encoder, the symbol are mapped and spreaded together. In other words, the input bits are directly mapped to multi-dimensional complex domain codewords selected from a predefined codebook set. Considering that designing optimal multi-dimensional sparse SCMA encoder is hard to solve, a multi-stage approach is proposed to obtain sub-optimal SCMA codebooks.

Let $\mathbf{c}$ be the $\mathrm{N}$-dimensional $(\mathrm{N}$ is the number of resources that each user occupies) complex constellation point defined in the mother constellation set $\mathcal{C} \subset \mathbb{C}^{N}$. And define the mapping from $\mathbb{B}^{\log _{2}^{M}}$ to $\mathcal{C}$ in (2).

$$
\mathbb{B}^{\log _{2}^{M}} \rightarrow \mathcal{C}, \mathbf{c}=g(\mathbf{b})
$$

Then the SCMA encoder in (1) can be written as follows.

$$
f: \equiv \mathbf{V} g, \mathbf{x}=\mathbf{V} g(\mathbf{b})
$$

where the binary mapping matrix $\mathbf{V} \in \mathbb{B}^{K \times N}$ maps $N$ dimensional mother constellation to $K$-dimensional codewords. The spreading matrix $\mathbf{V}$ includes $K-N$ all-zero rows, so all the codewords of SCMA codebook contain $K-N$ dimension zero-element. Eliminating the all-zero rows from $\mathbf{V}$, the rest can be represented by identity matrix $\mathbf{I}_{N}$. That is, the binary mapper does not permute the dimensions of subspace $\mathcal{C}$ during the mapping process. The codebook contains $M$ codewords each consisting of $K$ complex values from which no more than $N$ elements are non-zero specified by the matrix V.

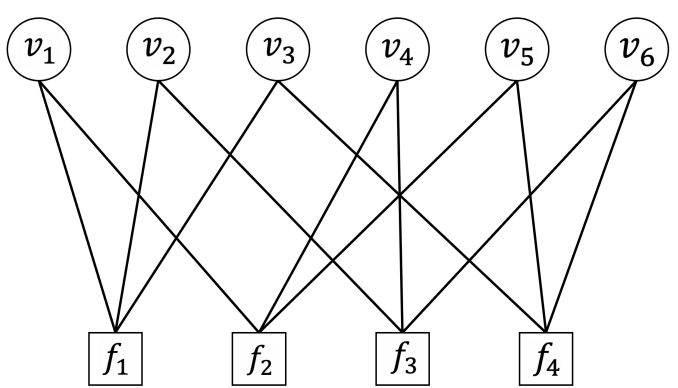

Fig. 2: Factor graph representation of an SCMA system with $J=6$, $K=4, N=2$ and $d_{f}=3$.

\section{A. Traditional Multi-users Codebook Design Method}

In traditional SCMA codebook design method proposed in [10] and used in most papers about SCMA codebook design, the codebook design method is sub-optimal [10] and the steps are similar.

The whole structure of SCMA codebook can be represented by a factor graph defined as $\mathbf{F}=\left(\mathbf{f}_{1}, \mathbf{f}_{2}, \cdots, \mathbf{f}_{J}\right)$, where $\mathbf{f}_{j}=\operatorname{diag}\left(\mathbf{V}_{j} \mathbf{V}_{j}^{T}\right), \mathbf{V}_{j}$ is the sparse matrix for user $j$. The layer node $(\mathrm{LN}) j$ and resource node $(\mathrm{RN}) k$ are connected only if $\mathbf{F}_{i j}=1$. Fig. 2 shows an example of a factor graph representation with $6 \mathrm{LNs}$ and $4 \mathrm{RNs}$, where each $\mathrm{LN}$ is connected with $N=2 \mathrm{RNs}$, and each $\mathrm{RN}$ is shared by $d_{f}=3$ LNs. The factor graph is as follows.

$$
\mathbf{F}=\left[\begin{array}{llllll}
1 & 1 & 1 & 0 & 0 & 0 \\
1 & 0 & 0 & 1 & 1 & 0 \\
0 & 1 & 0 & 1 & 0 & 1 \\
0 & 0 & 1 & 0 & 1 & 1
\end{array}\right]
$$

In order to design the SCMA codebook with the size of $K \times M \times J$, generally, the first step is to design a mother constellation for a user, whose size is $N \times M$. Then, for each user, handle the mother constellation with the constellation operator $\Delta$ and generate a matrix. Thirdly, expand the matrix to a sparse one with $\mathbf{V}_{j}$. Fig. 3 illustrates the process of SCMA codebook design intuitively. In this way, the size of codebook for each user expands from $N \times M$ to $K \times M$.

$$
\mathbf{C}_{j}=\mathbf{V}_{j}\left(\Delta_{j}\right) \mathbf{A}_{M C}
$$

where $\Delta_{j}$ is the constellation operator for user $j$ and $\mathbf{A}_{M C}$ is the mother constellation.

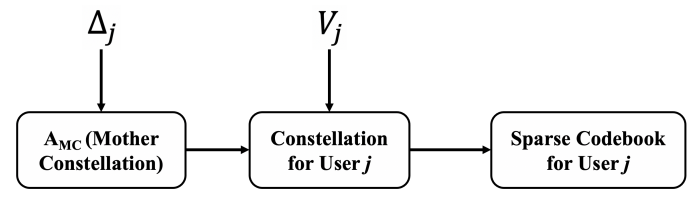

Fig. 3: Flow chart for traditional multi-users codebook design

\section{B. Multi-resources Codebook Design Method}

In this subsection, we propose a different structure to design the SCMA codebook. Redefine the factor graph as 
$\mathbf{F}=\left(\mathbf{f}_{1}, \mathbf{f}_{2}, \cdots, \mathbf{f}_{K}\right)^{T}$, where $\mathbf{f}_{k}=\operatorname{diag}\left(\mathbf{V}_{k} \mathbf{V}_{k}^{T}\right), \mathbf{V}_{k}$ is the sparse matrix for resource $k$. The steps to design a SCMA codebook are: 1). design the mother constellation with the size of $M \times d_{f}$ for a resource; 2). handle the mother constellation with the $k_{t h}$ resource's operator $\Delta_{k} ; 3$ ). expand the constellation for resource $k$ to a sparse matrix with $\mathbf{V}_{k}$.

$$
\mathbf{C}_{k}=\left(\Delta_{k}\right) \mathbf{A}_{M C} \mathbf{V}_{k}^{T}
$$

where $\Delta_{k}$ is the constellation operator for resource $k$.

The sparse matrix transforms $k_{t h}$ resource's constellation with the size of $M \times d_{f}$ to its codebook, whose size is $M \times J$. The process is shown in Fig. 4. It's worth noting that the multiresources structure is only used for codebook design not for the encoding process. The system structure in Fig. 1 is unchanged.

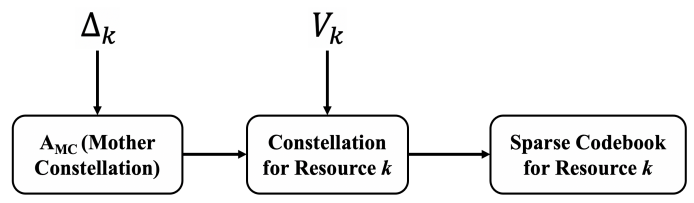

Fig. 4: Flow chart for multi-resources codebook design

\section{MS-SCMA CODEBook DeSIGN Method}

In this section, a design method for multi-resources mother constellation $A_{M C}$ based on the square-QAM signal constellation (MS-SCMA) is introduced and discussed. Then, SCMA codebooks are constructed with the mother constellation.

Compared with LDS, SCMA enjoys additional degree of freedom in multi-dimensional codebooks design, which brings extra shaping gain. The power variation for different users helps MPA receivers decrease interferences. Also, considering the orthogonality of $K$ resources, the constellations for different resources can use the same configuration, which means lower complexity for codebook design. The procedure of previous SCMA codebook design method is summarized as follows.

Step 1. Generate the sparse matrix $\mathbf{V}_{j}$ of user $j$ by inserting $K-N$ all-zero row vectors into the rows of $\mathbf{I}_{N}$, which comes from the factor graph;

Step 2. Generate the mother constellation $\mathbf{A}_{M C}$ with the size of $N \times M$;

Step 3. Determine the operator $\Delta_{j}$, and the codebook $\mathbf{C}_{j}$ for user $j$ is $\mathbf{C}_{j}=\mathbf{V}_{j}\left(\Delta_{j}\right) \mathbf{A}_{M C}$.

The method aims to design the constellations for each user by handling the mother constellation with the user operators. So the previous works focus on the design of mother constellation and user operators. The performance of SCMA codebook depends on the choice of mother constellation. Especially, the SCMA codebook is the same as the LDS codebook if the mother constellation is simply traditional modulations (e.g., PSK). And the user operators are usually the combination of typical operators, including phase rotation, matrix permutation and length extension [17]. (i). Phase rotation $(\circ: \phi) \mathbf{Z}:=e^{i \phi} \mathbf{Z}$

(ii). length extension $(*: \lambda) \mathbf{Z}:=\lambda \mathbf{Z}$

(iii). matrix permutation

$(\otimes: \mathbf{\Pi}) \mathbf{Z}:=\mathbf{\Pi Z}$.

Compared with LDS, SCMA enjoys additional degree of freedom in multi-dimensional codebooks design, which brings extra shaping gain. The power variation for different users helps MPA receivers decrease interferences. Also, considering the orthogonality of $K$ resources, the constellations for different resources can use the same configuration, which means lower complexity for codebook design.

The steps of MS-SCMA codebook design are illustrated in detail as follows.

The first step is to construct the mother constellation. In order to construct a mother constellation simply, first design an initial constellation of $M$ points. In this paper, we use $M$ point square-QAM, $\mathbf{a}_{0}$, which is the most simple constellation. Then, construct $d_{f}$ sub-constellations by handling the initial constellation with resources' operators $\Delta_{k},(k=1,2, \cdots, K)$.

$$
\begin{gathered}
\mathbf{a}_{1}=\left(* \circ: \lambda_{1} \phi_{1}\right) \mathbf{a}_{0}=\lambda_{1} \cdot e^{i \phi_{1}} \cdot \mathbf{a}_{0} \\
\mathbf{a}_{2}=\left(* \circ: \lambda_{2} \phi_{2}\right) \mathbf{a}_{0}=\lambda_{2} \cdot e^{i \phi_{2}} \cdot \mathbf{a}_{0} \\
\vdots \\
\mathbf{a}_{d_{f}}=\left(* \circ: \lambda_{d_{f}} \phi_{d_{f}}\right) \mathbf{a}_{0}=\lambda_{d_{f}} \cdot e^{i \phi_{d_{f}}} \cdot \mathbf{a}_{0}
\end{gathered}
$$

where $\lambda_{1}, \lambda_{2}, \cdots, \lambda_{d_{f}}$ are length extension operators and $\phi_{1}, \phi_{2}, \cdots, \phi_{d_{f}}$ are phase rotation operators.

The mother constellation can be obtained by the subconstellations with the size of $M \times d_{f}$.

$$
\mathbf{A}_{M C}=\left(\mathbf{a}_{1}, \mathbf{a}_{2}, \cdots, \mathbf{a}_{d_{f}}\right)
$$

The second step is to generate the sparse matrix. According to the known factor graph $\mathbf{F}$, insert $J-d_{f}$ all-zero row vectors into the rows of rows of $\mathbf{I}_{d_{f}}$ and generate the sparse matrix $\mathbf{V}_{k}$ for resource $k$.

And the last step is to construct SCMA codebook based on the mother constellation and sparse matrix. Considering that signals on different sources are independent, the constellations on different resources can be the same. So when designing the operators for different resources, length extension and phase rotation are not necessary. And in order to get extra gain, for one user, the constellations on different sources should be different. For the resource $k$, the codebook is designed as (8) shows.

$$
\mathbf{C}_{k}=\left(\Delta_{k}\right) \mathbf{A}_{M C} \mathbf{V}_{k}^{T}
$$

For user $k=1,2, \cdots, K$, the operator $\Delta_{k}$ can be designed as follows.

$$
\Delta_{k}=\left(\otimes: \boldsymbol{\Pi}_{k}\right)
$$

where $\Pi_{k}$ is a permutation matrix, which adjusts the order of $\mathbf{a}_{1}, \mathbf{a}_{2}, \cdots, \mathbf{a}_{d_{f}}$ in the mother constellation $\mathbf{A}_{M C}$. 
Notice that for one user, the power on different resources should be different and the power level of different users should vary.

Finally, generate the SCMA codebook based on the resources' codebooks.

$$
\mathbf{C B}=\left[\mathbf{C}_{1}, \mathbf{C}_{2}, \cdots, \mathbf{C}_{K}\right]^{T}
$$

To illustrate the process clearly, take the SCMA system with $6 \mathrm{LNs}$ and 4 RNs as an example to show how it works.

Assume that the SCMA codebook is quaternary modulation, $M=4$. Firstly, construct an initial constellation. In this paper, we use the most simple modulation, 4QAM, to design the mother constellation. The initial constellation is $\mathbf{a}_{0}=[-1,-i, 1, i]^{T}$.

Then, rotate and extend the initial constellation, so subconstellations can be obtained in Fig. 5 , in which $\mathbf{a}_{1}, \mathbf{a}_{2}, \mathbf{a}_{3}$ are sub-constellation 1, sub-constellation 2 and sub-constellation 3 respectively.

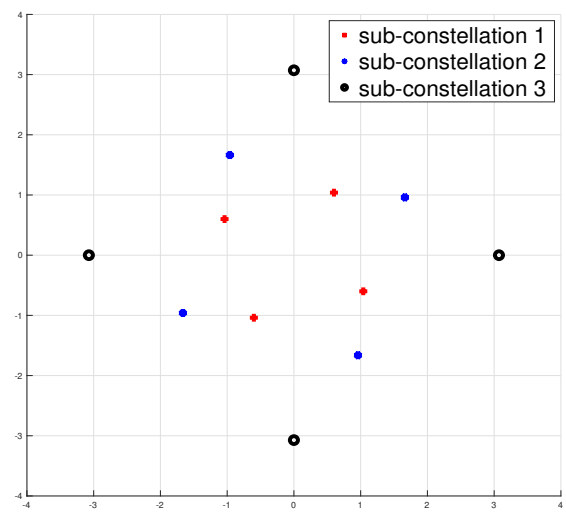

Fig. 5: The initial constellation for quaternary modulation SCMA codebook

And Fig. 2 is the factor graph of the system. According to the steps in the above, the SCMA codebook can be represented by matrix (11).

$$
\mathbf{C B}=\left[\begin{array}{cccccc}
\mathbf{a}_{1} & \mathbf{a}_{2} & \mathbf{a}_{3} & 0 & 0 & 0 \\
\mathbf{a}_{2} & 0 & 0 & \mathbf{a}_{3} & \mathbf{a}_{1} & 0 \\
0 & \mathbf{a}_{3} & 0 & \mathbf{a}_{2} & 0 & \mathbf{a}_{1} \\
0 & 0 & \mathbf{a}_{1} & 0 & \mathbf{a}_{3} & \mathbf{a}_{2}
\end{array}\right]
$$

\section{SCMA CODEBOOK DESIGN CRITERIA}

In this section, we aim to find effective criteria to measure the performance of SCMA codebooks in Rayleigh fading channel and AWGN channel respectively. A good codebook means the SCMA system has low bit error rate (BER). So in this paper, we need to estimate the error probability of the SCMA system to find the optimization criteria.

According to [19] and [20], to obtain a low error probability at a given signal-to-noise ratio $E_{b} / N_{0}$, the minimum Euclidean distance $d_{E_{m i n}}$ (the nearest distance between any two points in the constellation) should be maximized while the kissing number (the number of nearest-two-points pairs) $\tau$ should be minimized.

So assuming that the average energy per constellation point is set, the optimization function is in (12).

$$
\varphi=\frac{\tau}{d_{E_{\text {min }}}}
$$

For the Rayleigh fading channel, the estimation of pairwise error probability can be given by the standard Chernoff bound technique [21] or the direct calculation with the Gaussian tail function approximation [20]. Different from that in AWGN channel, the product distance is considered instead of the Euclidean distance for Rayleigh fading channel.

For SCMA codebooks, assuming that the average energy per constellation point is constant, the minimum product distance should be maximized while the kissing number is minimized. So the optimization function is as follows.

$$
\varphi=\frac{\tau}{\min \left(d_{p}^{(L)}\left(\mathbf{x}_{1}, \mathbf{x}_{2}\right)\right)}
$$

\section{MS-SCMA Codebook Optimization Design BASED on Simulated Annealing Algorithm}

According to the criteria proposed in Section IV, the problem of SCMA codebooks design converts to minimize the criteria function (12) and (13), which is an optimization problem.
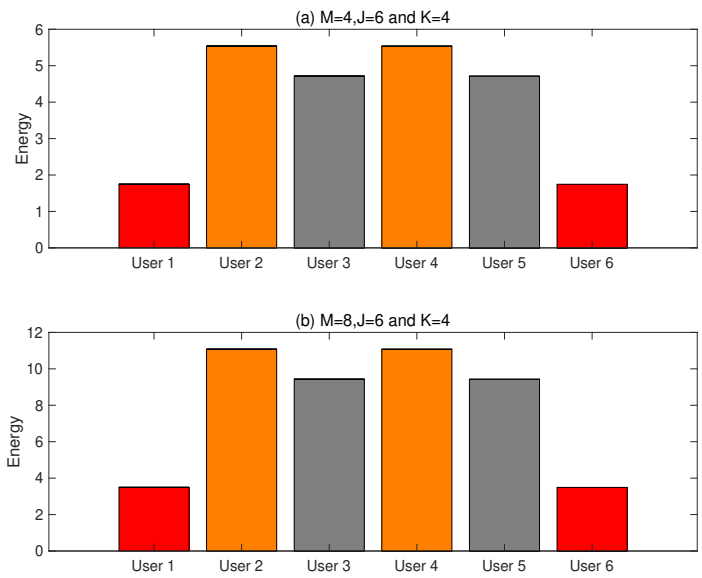

Fig. 6: Energy for Users

In this paper, we choose simulated annealing algorithm, a classical meta-heuristics algorithm, to optimize the criteria function. As a technically-mature optimization algorithm, simulated annealing algorithm can solve large-scale problem in a reasonable time cost and jump out of the local optimum with a certain probability to find the global optimum [22, 23].

The $M$-point SCMA codebook can be designed by the MSSCMA method based on QAM constellations, in which the parameters $\lambda_{1}, \lambda_{2}, \cdots, \lambda_{d_{f}}, \phi_{1}, \phi_{2}, \cdots, \phi_{d_{f}}$ are supposed to be optimized according to the criteria in (12) and (13). 
Fig. 6 shows the energy for each user, when there are 6 users and 4 resources. Fig. 7 and Fig. 8 show the simulation BER results of the SCMA codebooks designed by MS-SCMA, star-QAM SCMA codebooks in [15] and GAM codebooks in [3], For Rayleigh fading channel, compared with SCMA codebook design method based on GAM and star-QAM, the BER performance of MS-SCMA codebook design method increases by about $8 \mathrm{~dB}$. And for AWGN channel, it also brings some improvement, especially for high-order SCMA codebook.

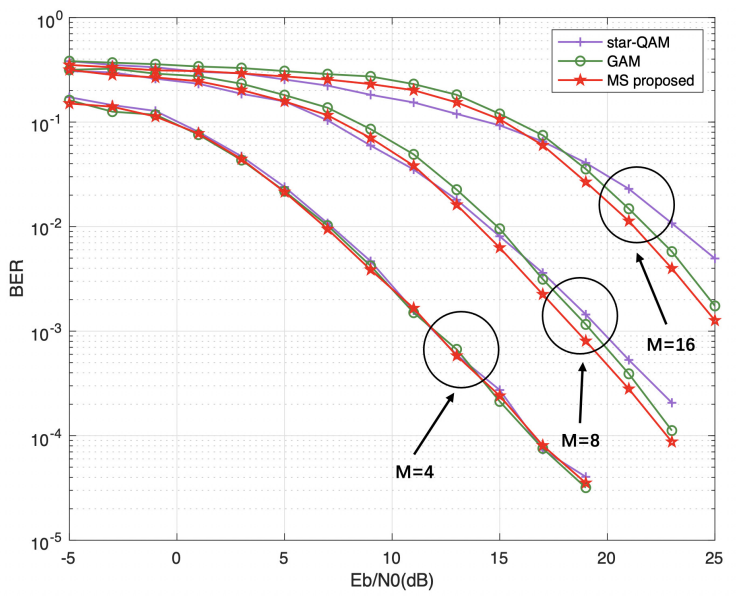

Fig. 7: BER performance in AWGN channel when $\mathrm{K}=4$ and $\mathrm{J}=6$

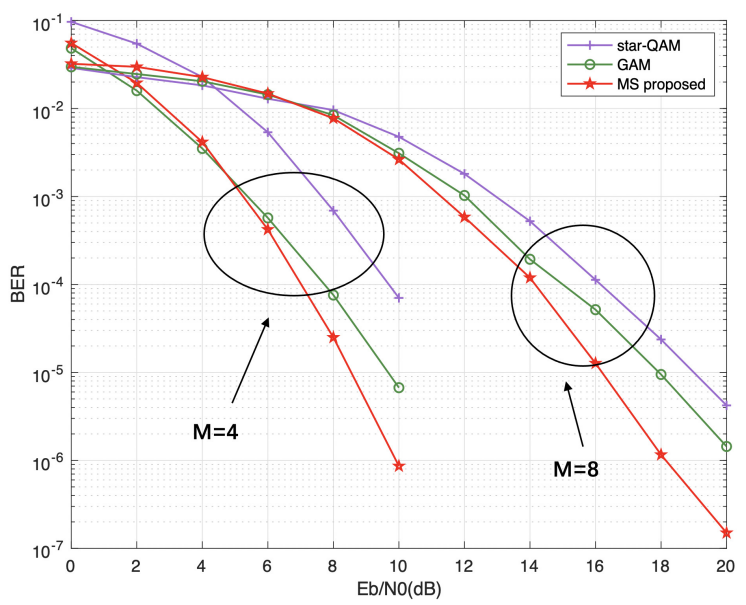

Fig. 8: BER performance in Rayleigh fading channel when $\mathrm{K}=4$ and $\mathrm{J}=6$

\section{CONCLUSION}

In this paper, we have proposed a new class of SCMA codebook which displays power imbalance among different users. Simulation results have shown that our proposed MS codebook offers substantial error rate gains over several known codebooks in Gaussian channel and Rayleigh fading channel. The gain stems from the fact that the power imbalance of the proposed codebooks helps to optimize the criteria which is useful for enhanced interference cancellation in MPA decoding.

\section{REFERENCES}

[1] M. J. Marcus, "5G and "IMT for 2020 and beyond" [Spectrum Policy and Regulatory Issues]," IEEE Wireless Communications, vol. 22, pp. 23, August 2015.

[2] A. Benjebbour, Y. Saito, Y. Kishiyama, A. Li, A. Harada, and T. Nakamura, "Concept and practical considerations of non-orthogonal multiple access (NOMA) for future radio access," in 2013 International Symposium on Intelligent Signal Processing and Communication Systems, pp. 770-774, Nov 2013.

[3] Z. Mheich, L. Wen, P. Xiao, and A. Maaref, "Design of SCMA Codebooks Based on Golden Angle Modulation," IEEE Transactions on Vehicular Technology, vol. 68, pp. 1501-1509, Feb 2019.

[4] S. M. R. Islam, N. Avazov, O. A. Dobre, and K. Kwak, "PowerDomain Non-Orthogonal Multiple Access (NOMA) in 5G Systems: Potentials and Challenges," IEEE Communications Surveys Tutorials, vol. 19, pp. 721-742, Secondquarter 2017.

[5] Q. Luo, P. Gao, Z. Liu, L. Xiao, Z. Mheich, P. Xiao, and A. Maaref, "An error rate comparison of power domain non-orthogonal multiple access and sparse code multiple access," IEEE Open Journal of the Communications Society, vol. 2, pp. 500-511, 2021.

[6] R. Hoshyar, F. P. Wathan, and R. Tafazolli, "Novel Low-Density Signature for Synchronous CDMA Systems Over AWGN Channel," IEEE Transactions on Signal Processing, vol. 56, pp. 1616-1626, April 2008.

[7] R. Hoshyar, R. Razavi, and M. Al-Imari, "LDS-OFDM an Efficient Multiple Access Technique," in 2010 IEEE 71st Vehicular Technology Conference, pp. 1-5, May 2010.

[8] X. Li, Z. Gao, Z. Liu, P. Xiao, and Y. Gui, "Design of power-imbalanced scma codebook," 2020.

[9] J. Dai, K. Niu, and J. Lin, "Code-Domain Non-Orthogonal Multiple Access for Visible Light Communications," in 2018 IEEE Globecom Workshops (GC Wkshps), pp. 1-6, Dec 2018.

[10] M. Taherzadeh, H. Nikopour, A. Bayesteh, and H. Baligh, "SCMA Codebook Design," in 2014 IEEE 80th Vehicular Technology Conference (VTC2014-Fall), pp. 1-5, Sep. 2014.

[11] J. van de Beek and B. M. Popovic, "Multiple Access with Low-Density Signatures," in GLOBECOM 2009 - 2009 IEEE Global Telecommunications Conference, pp. 1-6, Nov 2009.

[12] Z. Liu and L. L. Yang, "Sparse or dense: A comparative study of codedomain noma systems," IEEE Transactions on Wireless Communications, pp. 1-1, 2021.

[13] H. Nikopour and H. Baligh, "Sparse code multiple access," in 2013 IEEE 24th Annual International Symposium on Personal, Indoor, and Mobile Radio Communications (PIMRC), pp. 332-336, Sep. 2013.

[14] D. Cai, P. Fan, X. Lei, Y. Liu, and D. Chen, "Multi-Dimensional SCMA Codebook Design Based on Constellation Rotation and Interleaving," in 2016 IEEE 83rd Vehicular Technology Conference (VTC Spring), pp. 15, May 2016.

[15] L. Yu, X. Lei, P. Fan, and D. Chen, "An optimized design of SCMA codebook based on star-QAM signaling constellations," in 2015 International Conference on Wireless Communications Signal Processing (WCSP), pp. 1-5, Oct 2015.

[16] Y. Zhou, Q. Yu, W. Meng, and C. Li, "SCMA codebook design based on constellation rotation," in 2017 IEEE International Conference on Communications (ICC), pp. 1-6, May 2017.

[17] H. Yan, H. Zhao, Z. Lv, and H. Yang, "A top-down SCMA codebook design scheme based on lattice theory," in 2016 IEEE 27th Annual International Symposium on Personal, Indoor, and Mobile Radio Communications (PIMRC), pp. 1-5, Sep. 2016.

[18] Z. Mheich, Z. Liu, P. Xiao, and A. Maaref, "Delayed bit interleaved coded sparse code multiple access," IEEE Transactions on Vehicular Technology, vol. 69, no. 7, pp. 8018-8022, 2020.

[19] J. H. Conway and N. J. A. Sloane, Sphere Packings, Lattices and Groups. New York, NY: Springer, 1999.

[20] J. Boutros, E. Viterbo, C. Rastello, and J. . Belfiore, "Good lattice constellations for both Rayleigh fading and Gaussian channels," IEEE Transactions on Information Theory, vol. 42, pp. 502-518, March 1996.

[21] D. Divsalar and M. K. Simon, "The design of trellis coded MPSK for fading channels: set partitioning for optimum code design," IEEE Transactions on Communications, vol. 36, pp. 1013-1021, Sep. 1988.

[22] E. Besnard, N. Cordier-Lallouet, O. Kural, and H. Chen, Design/optimization with advanced simulated annealing.

[23] E. H. L. A. Peter J. M. van Laarhoven, Simulated Annealing: Theory and Applications. Springer, Dordrecht, Aug 1987. 